\title{
Positive Schatten class Toeplitz operators on the ball
}

\author{
by \\ Boo Rim Choe (Seoul), Hyungwoon Koo (Seoul) \\ and Young Joo Lee (Gwangju)
}

\begin{abstract}
On the harmonic Bergman space of the ball, we give characterizations for an arbitrary positive Toeplitz operator to be a Schatten class operator in terms of averaging functions and Berezin transforms.
\end{abstract}

1. Introduction. For a fixed integer $n \geq 2$, let $B=B_{n}$ denote the open unit ball in $\mathbb{R}^{n}$. The harmonic Bergman space $b^{2}=b^{2}(B)$ is the set of all complex-valued harmonic functions $f$ on $B$ such that

$$
\|f\|_{2}=\left\{\int_{B}|f|^{2} d V\right\}^{1 / 2}<\infty,
$$

where $V$ denotes the Lebesgue volume measure on $B$. For simplicity, we use the notation $d y=d V(y)$, etc.

For $0<p \leq \infty$, let $L^{p}=L^{p}(V)$ be the Lebesgue spaces on $B$. As is well known, $b^{2}$ is a closed subspace of $L^{2}$ and hence is a Hilbert space. By the mean value property of harmonic functions, it is easily seen that point evaluations are continuous on $b^{2}$. Thus, to each $x \in B$, there corresponds a unique $R(x, \cdot) \in b^{2}$ which has the following reproducing property:

$$
f(x)=\int_{B} f(y) \overline{R(x, y)} d y, \quad x \in B,
$$

for all $f \in b^{2}$. The explicit formula for the kernel function $R(x, y)$ is well known:

$$
R(x, y)=\frac{1}{|B|} \cdot \frac{1}{[x, y]^{n}}\left\{\left(\frac{1-|x|^{2}|y|^{2}}{[x, y]}\right)^{2}-\frac{4|x|^{2}|y|^{2}}{n}\right\}
$$

for $x, y \in B$, where $[x, y]=\sqrt{1-2 x \cdot y+|x|^{2}|y|^{2}}$. Here, as elsewhere, we write $x \cdot y$ for the dot product of $x, y \in \mathbb{R}^{n}$ and $|E|=V(E)$ for the volume

2000 Mathematics Subject Classification: Primary 47B35; Secondary 31B05.

Key words and phrases: Toeplitz operator, harmonic Bergman space, Schatten class. This research was supported by KOSEF(R01-2003-000-10243-0). 
of Borel sets $E \subset B$. Hence the kernel function $R(x, y)$ is real and hence the complex conjugation in the integral of (1.1) can be removed. See [2] for more information and related facts.

Let $R$ be the Hilbert space orthogonal projection from $L^{2}$ onto $b^{2}$. The reproducing property (1.1) yields the following integral representation of $R$ :

$$
R \psi(x)=\int_{B} \psi(y) R(x, y) d y, \quad x \in B,
$$

for functions $\psi \in L^{2}$. It is easily seen that the projection $R$ can be extended to an integral operator via (1.3) from $L^{1}$ into the space of all harmonic functions on $B$. It even extends to $\mathcal{M}$, the space of all complex Borel measures on $B$. Namely, for each $\mu \in \mathcal{M}$, the integral

$$
R \mu(x)=\int_{B} R(x, y) d \mu(y), \quad x \in B,
$$

defines a function harmonic on $B$. For $\mu \in \mathcal{M}$, the Toeplitz operator $T_{\mu}$ with symbol $\mu$ is defined by

$$
T_{\mu} f=R(f d \mu)
$$

for $f \in b^{2} \cap L^{\infty}$. Note that $T_{\mu}$ is defined on a dense subset of $b^{2}$, because the bounded harmonic functions form a dense subset of $b^{2}$.

A Toeplitz operator $T_{\mu}$ is called positive if $\mu$ is a positive finite Borel measure (hereafter we simply write $\mu \geq 0$ ). For positive Toeplitz operators on harmonic Bergman spaces, basic operator-theoretic properties such as boundedness, compactness and the membership in the Schatten classes $S_{p}$ (see Section 4), $1 \leq p<\infty$, have been studied in various settings; see [7], [11], [12] and references therein. In this paper we extend the characterization in [7] for Schatten class positive Toeplitz operators to the full range $0<p<\infty$.

To state our result we briefly introduce some notation. Given $\mu \geq 0$, $\widehat{\mu}_{r}$ denotes the averaging function over pseudohyperbolic balls with radius $r$, and $\widetilde{\mu}$ denotes the Berezin transform. See Section 3 for relevant definitions. Also, we let $\lambda$ denote the measure on $B$ defined by

$$
d \lambda(x)=\left(1-|x|^{2}\right)^{-n} d x .
$$

The next theorem is the main result of this paper. In the case $1 \leq p<\infty$, that theorem (with slightly different averaging functions) has been proved in [7]. In the case $0<p<1$, extending results in [15] for holomorphic Bergman spaces to the harmonic case, the authors [4] have obtained the corresponding results on the harmonic Bergman space of the upper halfspace. While the main scheme of our proof is adapted from [4] or [15], we need to establish corresponding theorems for the harmonic Bergman space on the ball. The cut-off point $(n-1) / n$ is sharp in the theorem below. 
TheOREM 1.1. Let $0<p<\infty, 0<r<1$ and $\mu \geq 0$. Then the following two statements are equivalent:

(a) $T_{\mu} \in S_{p}$.

(b) $\widehat{\mu}_{r} \in L^{p}(\lambda)$.

Moreover, if $(n-1) / n<p<\infty$, then the above statements are also equivalent to

(c) $\widetilde{\mu} \in L^{p}(\lambda)$.

In Section 2 we recall some basic properties of Möbius transformations, and collect some basic estimates for the pseudohyperbolic distance and the kernel function. In Section 3 we investigate weighted $L^{p}$-behavior of averaging functions and Berezin transforms. In Section 4 we prove Theorem 1.1. In Section 5 we provide examples indicating that the parameter ranges required for all the results of the previous sections are best possible.

Constants. In the rest of the paper the same letter $C$ will denote various positive constants, unless otherwise specified, which may change at each occurrence. The constant $C$ may often depend on the dimension $n$ and some other parameters like $\delta, p, k$, or $r$, etc., but it will always be independent of particular functions, measures, points or sequences under consideration. We will often abbreviate inessential constants involved in inequalities by writing $X \lesssim Y$ or $Y \gtrsim X$ for positive quantities $X$ and $Y$ if the ratio $X / Y$ has a positive upper bound. Also, we write $X \approx Y$ if $X \lesssim Y$ and $X \gtrsim Y$.

2. Preliminaries. In this section we introduce notation and collect several basic lemmas which will be used in later sections.

2.1. Möbius transformation. We first recall Möbius transformations on $B$. All relevant details can be found in [1, pp. 17-30]. Let $a \in B$. The canonical Möbius transformation $\phi_{a}$ that exchanges $a$ and 0 is given by

$$
\phi_{a}(x)=a+\left(1-|a|^{2}\right)\left(a-x^{*}\right)^{*}
$$

for $x \in B$ (note $\phi_{a}=-T_{a}$ in the notation of [1]). Here $x^{*}=x /|x|^{2}$ denotes the inversion of $x$ with respect to the sphere $\partial B$. Avoiding the $x^{*}$ notation, we have

$$
\phi_{a}(x)=\frac{\left(1-|a|^{2}\right)(a-x)+|a-x|^{2} a}{[x, a]^{2}} .
$$

The map $\phi_{a}$ is an involution of $B$, i.e., $\phi_{a}^{-1}=\phi_{a}$. We will tacitly use the following identities:

$$
\begin{aligned}
\left|\phi_{a}(x)\right| & =\frac{|x-a|}{[x, a]}, & {\left[\phi_{a}(x), a\right] } & =\frac{1-|a|^{2}}{[x, a]}, \\
1-\left|\phi_{a}(x)\right|^{2} & =\frac{\left(1-|x|^{2}\right)\left(1-|a|^{2}\right)}{[x, a]^{2}}, & J \phi_{a}(x) & =\left(\frac{1-|a|^{2}}{[x, a]^{2}}\right)^{n},
\end{aligned}
$$


where $J \phi_{a}$ denotes the Jacobian of $\phi_{a}$. Also, using these identities, one may easily verify that the measure $\lambda$ is Möbius invariant.

2.2. Pseudohyperbolic distance. The hyperbolic distance $\beta(x, y)$ between two points $x, y \in B$ is given by

$$
\beta(x, y)=\frac{1}{2} \log \frac{1+\left|\phi_{y}(x)\right|}{1-\left|\phi_{y}(x)\right|} .
$$

As is well known, $\beta$ is Möbius invariant. Let $\varrho(x, y)=\left|\phi_{y}(x)\right|$. Then $\varrho$ is also a Möbius invariant distance on $B$. We shall work with this pseudohyperbolic distance $\varrho$.

For $a \in B$ and $r \in(0,1)$, let $E_{r}(a)$ denote the pseudohyperbolic ball with radius $r$ and center $a$. A straightforward calculation shows that $E_{r}(a)$ is a Euclidean ball with

$$
\text { center }=\frac{\left(1-r^{2}\right)}{1-|a|^{2} r^{2}} a \quad \text { and } \quad \text { radius }=\frac{\left(1-|a|^{2}\right) r}{1-|a|^{2} r^{2}} .
$$

A straightforward calculation gives

$$
\inf _{x \in E_{r}(a)}(1-|x|)=\frac{(1-|a|)(1-r)}{1+|a| r} .
$$

This yields the following observation.

Lemma 2.1. The inequality

$$
\frac{1-\varrho(z, w)}{1+\varrho(z, w)} \leq \frac{1-|z|}{1-|w|} \leq \frac{1+\varrho(z, w)}{1-\varrho(z, w)}
$$

holds for $z, w \in B$.

We also have the following result.

LEMma 2.2. The inequality

$$
\frac{1-\varrho(z, w)}{1+\varrho(z, w)} \leq \frac{[z, a]}{[w, a]} \leq \frac{1+\varrho(z, w)}{1-\varrho(z, w)}
$$

holds for $z, w, a \in B$.

Proof. Let $z, w, a \in B$ and assume $\varrho(z, w)<r$. We may assume $a \neq 0$. Using (2.2), we note that $E_{r}(w)$ is contained in the Euclidean ball with center $w$ and radius $s$, where

$$
s=\frac{r\left(1-|w|^{2}\right)}{1-|w| r}<\frac{2 r(1-|w|)}{1-r}<\frac{2 r\left|w-a^{*}\right|}{1-r} .
$$

It follows that

$$
\left|z-a^{*}\right| \leq\left|w-a^{*}\right|+|z-w|<\frac{1+r}{1-r}\left|w-a^{*}\right|
$$


and hence

$$
\frac{[z, a]}{[w, a]}=\frac{|a|\left|z-a^{*}\right|}{|a|\left|w-a^{*}\right|}=\frac{\left|z-a^{*}\right|}{\left|w-a^{*}\right|}<\frac{1+r}{1-r} .
$$

This implies the lemma.

Note that by (2.2) we have

$$
\left|E_{r}(a)\right|=|B|\left(\frac{\left(1-|a|^{2}\right) r}{1-|a|^{2} r^{2}}\right)^{n} .
$$

Consequently, we obtain by Lemma 2.1 an estimate on the size of pseudohyperbolic balls: Given $\delta, t \in(0,1)$, there is a constant $C=C(\delta, t, n)>0$ such that

$$
C^{-1} \leq \frac{\left|E_{r_{1}}(z)\right|}{\left|E_{r_{2}}(w)\right|} \leq C, \quad w \in E_{r_{3}}(z),
$$

whenever $r_{1}, r_{2}, r_{3}<\delta$ and $t<r_{1} / r_{2}<t^{-1}$.

2.3. Kernel estimates. Given an integer $k \geq 0$, we let $R_{k}(x, y)$ be the reproducing kernel for the weighted harmonic Bergman space with respect to the weight $(1-|x|)^{k}$. So, $R_{0}=R$ is the harmonic Bergman kernel mentioned before. Explicit formulas for these kernels are given in [8, (3.1)]. Note that $R_{k}(x, y)$ is real-valued and $R_{k}(x, y)=R_{k}(y, x)$ for every $x, y \in B$. We have the following estimates of these kernels:

$$
\begin{aligned}
& \left|R_{k}(x, x)\right| \approx \frac{1}{(1-|x|)^{n+k}} \text { and }\left|R_{k}(x, y)\right| \lesssim \frac{1}{[x, y]^{n+k}}, \\
& \left|\nabla_{y} R_{k}(x, y)\right| \lesssim \frac{1}{[x, y]^{n+k+1}}
\end{aligned}
$$

for all $x, y \in B$. Here and in what follows, $\nabla_{y}$ denotes the gradient with respect to the $y$-variable. For the second estimate of (2.6), see [8, Chapter 3]. Also, one can verify the first estimate for (2.6) by using the explicit formula for $R_{k}$ given in [8, Chapter 3] (or see [11, Proposition 4] for similar estimates with slightly different weighted reproducing kernels).

LEMma 2.3. Given an integer $k \geq 0$, there exist some $r_{k}=r_{k}(n) \in(0,1)$ and a constant $C=C(n, k)$ such that

$$
C^{-1} \leq R_{k}(x, y)(1-|x|)^{n+k} \leq C
$$

whenever $y \in E_{r}(x)$ and $0<r \leq r_{k}$.

Proof. By (2.6) we only need to prove the lower estimate. Let $x \in B$ and $y \in E_{r}(x)$ where $r$ is to be chosen later. Since $y \in E_{r}(x)$, by $(2.6)$ and 
Lemma 2.2 we have

$$
\begin{aligned}
R_{k}(x, x)-R_{k}(x, y) & \leq|y-x| \sup _{z \in E_{r}(x)}\left|\nabla_{z} R_{k}(x, z)\right| \lesssim r[x, y] \sup _{z \in E_{r}(x)} \frac{1}{[x, z]^{n+k+1}} \\
& <\frac{r}{(1-|x|)^{n+k}} \sup _{z \in E_{r}(x)} \frac{[x, y]}{[x, z]}<\frac{r}{(1-|x|)^{n+k}}\left(\frac{1+r}{1-r}\right)^{2} .
\end{aligned}
$$

Now, since $R_{k}(x, x) \approx(1-|x|)^{-n-k}$ by $(2.6)$, we have

$$
R_{k}(x, y) \gtrsim \frac{1}{(1-|x|)^{n+k}}\left[1-C r\left(\frac{1+r}{1-r}\right)^{2}\right]
$$

for some constant $C=C(n, k)>0$. Accordingly, taking $r=r_{k}$ small enough, we obtain the desired lower estimate.

In what follows, $d S$ denotes the surface area measure on $\partial B$.

LEMMA 2.4. Given c real, the following estimates hold:

$$
\int_{\partial B} \frac{d S(\zeta)}{|x-\zeta|^{n-1+c}} \approx \begin{cases}\left(1-|x|^{2}\right)^{-c} & \text { if } c>0 \\ 1+\left|\log \left(1-|x|^{2}\right)\right| & \text { if } c=0 \\ 1 & \text { if } c<0\end{cases}
$$

for $x \in B$. The constants suppressed above are independent of $x$.

Proof. Let $x \in B$ and denote the integral under consideration by $J_{c}(x)$. We will actually prove that the ratios of $J_{c}(x)$ and quantities in the desired estimates converge to a positive finite limit as $|x| \rightarrow 1$. Note that if $x=|x| \eta$ with $\eta \in S$, then $|x-\zeta|^{2}=(1-|x|)^{2}+2|x|(1-\eta \cdot \zeta)$ for $\zeta \in S$. Thus, using the slice integration formula (see $[2$, Corollary A.5]) and making a change of variables, we have

$$
\begin{aligned}
J_{c}(x) & =c_{n} \int_{-1}^{1} \frac{\left(1-r^{2}\right)^{(n-3) / 2}}{\left[(1-|x|)^{2}+2|x|(1-r)\right]^{(n-1+c) / 2}} d r \\
& =c_{n} \int_{0}^{2} \frac{[t(2-t)]^{(n-3) / 2}}{\left[(1-|x|)^{2}+2|x| t\right]^{(n-1+c) / 2}} d t
\end{aligned}
$$

for some constant $c_{n}$ depending only on $n$. It is clear from this that $J_{c}(x)$ converges to a positive finite limit as $|x| \rightarrow 1$ for $c<0$. Now, assume $c \geq 0$. Making another change of variables, we have

$$
\begin{aligned}
J_{c}(x)= & c_{n}(1-|x|)^{-c}(2|x|)^{-(n-1) / 2} \\
& \times \int_{0}^{4|x| /(1-|x|)^{2}}\left[2-\frac{(1-|x|)^{2} s}{2|x|}\right]^{(n-3) / 2} \frac{s^{(n-3) / 2}}{(1+s)^{(n-1+c) / 2}} d s
\end{aligned}
$$

for $x \neq 0$. It is now easily seen that $J_{c}(x)\left(1-|x|^{2}\right)^{c}$ converges to a positive 
finite limit as $|x| \rightarrow 1$ for $c>0$. Also, one can show that $J_{0}(x) \log \left(1-|x|^{2}\right)^{-1}$ converges to a positive finite limit as $|x| \rightarrow 1$.

We need to estimate the integrals $I_{c, \alpha}$ defined for $\alpha>-1$ and $c$ real as follows:

$$
I_{c, \alpha}(x)=\int_{B} \frac{\left(1-|y|^{2}\right)^{\alpha}}{[x, y]^{n+\alpha+c}} d y, \quad x \in B .
$$

The following estimate is proved in [13, Lemma 4.2]. Here we provide another proof by means of Lemma 2.4 for the reader's convenience.

Lemma 2.5. Let $\alpha>-1$ and $c$ be any real number. Then

$$
I_{c, \alpha}(x) \approx \begin{cases}\left(1-|x|^{2}\right)^{-c} & \text { if } c>0 \\ 1-\log \left(1-|x|^{2}\right) & \text { if } c=0 \\ 1 & \text { if } c<0\end{cases}
$$

for $x \in B$. The constants suppressed above are independent of $x$.

Proof. Let $x \in B$. We may assume $|x|>1 / 2$. Since $[y, \zeta]=|y-\zeta|$ for $y \in B$ and $\zeta \in \partial B$, integrating in polar coordinates yields

$$
I_{c, \alpha}(x)=n|B| \int_{0}^{1} \int_{\partial B} \frac{d S(\zeta)}{|r x-\zeta|^{n+\alpha+c}} r^{n-1}\left(1-r^{2}\right)^{\alpha} d r
$$

and thus the estimate for $\alpha+c \leq-1$ is clear by Lemma 2.4. So, we assume $\alpha+c>-1$. By Lemma 2.4 we have

$$
I_{c, \alpha}(x) \approx \int_{0}^{1} \frac{(1-r)^{\alpha}}{(1-r|x|)^{1+\alpha+c}} d r \approx\left(\int_{|x|}^{1}+\int_{0}^{|x|}\right) \frac{(1-r)^{\alpha}}{(1-r|x|)^{1+\alpha+c}} d r=I+I I .
$$

Note that $1-|x| \leq 1-r|x| \leq 2(1-|x|)$ for $|x| \leq r<1$. It follows that

$$
I \approx(1-|x|)^{-1-\alpha-c} \int_{0}^{1-|x|} r^{\alpha} d r \approx(1-|x|)^{-c} .
$$

Meanwhile, since $1-r \leq 1-r|x| \leq 2(1-r)$ for $0<r<|x|$, we have

$$
I I \approx \int_{0}^{|x|} \frac{d r}{(1-r)^{1+c}} \approx \int_{1-|x|}^{1} \frac{d r}{r^{1+c}}
$$

and thus

$$
I I \approx \begin{cases}(1-|x|)^{-c} & \text { if } c>0 \\ -\log (1-|x|) & \text { if } c=0 \\ 1 & \text { if } c<0 .\end{cases}
$$

Now, the lemma follows from the above estimates for $I$ and $I I$. 
3. Averaging functions and Berezin transform. In this section we collect the weighted $L^{p}$-behavior of the averaging function (as well as its discretized version) and of the Berezin transform.

Given $\mu \geq 0$ and $r \in(0,1)$, the averaging function $\widehat{\mu}_{r}$ and the Berezin transform $\widetilde{\mu}$ are defined by

$$
\widehat{\mu}_{r}(x)=\frac{\mu\left[E_{r}(x)\right]}{\left|E_{r}(x)\right|} \text { and } \widetilde{\mu}(x)=\left(1-|x|^{2}\right)^{n} \int_{B}|R(x, y)|^{2} d \mu(y)
$$

for $x \in B$. While it is customary to put $R(x, x)^{-1}$ in place of $\left(1-|x|^{2}\right)^{n}$ in the definition of the Berezin transform, we adopted the above definition for simplicity. For measurable functions $f$, we define $\widehat{f}_{r}$ and $\widetilde{f}$ similarly, whenever they are well defined.

Given $\alpha$ real, we let $L_{\alpha}^{p}=L^{p}\left(V_{\alpha}\right)$, where $V_{\alpha}$ denotes the weighted measure defined by $d V_{\alpha}(x)=\left(1-|x|^{2}\right)^{\alpha} d x$. For $\alpha=0$, we have $L_{0}^{p}=L^{p}$. Note $\lambda=V_{-n}$. Given $r \in(0,1)$, it is easily seen from (2.4) and Lemma 2.1 that

$$
V_{\alpha}\left[E_{r}(x)\right] \approx\left(1-|x|^{2}\right)^{n+\alpha}
$$

for $x \in B$. Also, given a sequence $\mathfrak{a}=\left\{a_{m}\right\}$ in $B$, we let $\ell^{p, \alpha}(\mathfrak{a})$ denote the $p$-summable sequence space weighted by $\left\{\left(1-\left|a_{m}\right|^{2}\right)^{\alpha}\right\}$. For $\alpha=0$, we let $\ell^{p}=\ell^{p, 0}(\mathfrak{a})$.

To obtain the weighted $L^{p}$-behavior of averaging functions and of the Berezin transform, we need to prove several preliminary results. We begin with a simple observation which in particular shows that averaging functions, when the radii are small enough, are dominated by the Berezin transform.

LEMMA 3.1. Let $k \geq 0$ be an integer, $\mu \geq 0$ and $0<r \leq r_{k}$, where $r_{k}$ is the number provided by Lemma 2.3. Then there exists a constant $C=$ $C(n, k, r)$ such that

$$
\widehat{\mu}_{r}(a) \leq C\left(1-|a|^{2}\right)^{n+2 k} \int_{B}\left|R_{k}(x, a)\right|^{2} d \mu(x), \quad a \in B .
$$

In particular, $\widehat{\mu}_{r} \leq C \widetilde{\mu}$ for $0<r \leq r_{0}$.

Proof. Let $a \in B$ be given. By Lemma 2.3 and (2.4), we have

$$
\int_{E_{r}(a)}\left|R_{k}(x, a)\right|^{2} d \mu(x) \gtrsim \frac{\mu\left[E_{r}(a)\right]}{\left(1-|a|^{2}\right)^{2(n+k)}} \approx \frac{\widehat{\mu}_{r}(a)}{\left(1-|a|^{2}\right)^{n+2 k}}
$$

where the constants suppressed depend only on $n, k$ and $r$. This implies the first part of the lemma. The second part is clear (with $k=0$ ).

Let $\left\{a_{m}\right\}$ be a sequence in $B$ and $r \in(0,1)$. We say that $\left\{a_{m}\right\}$ is $r$ separated if the balls $E_{r}\left(a_{m}\right)$ are pairwise disjoint, or simply say that $\left\{a_{m}\right\}$ is separated if it is $r$-separated for some $r$. Also, we say that $\left\{a_{m}\right\}$ is an $r$-lattice if it is $r / 2$-separated and $B=\bigcup_{m} E_{r}\left(a_{m}\right)$. One can explicitly construct an 
$r$-lattice by using the same argument as in [8]. Note that any "maximal" $r / 2$-separated sequence is an $r$-lattice.

The next two lemmas are basic properties involving separated sequences we use later. The first lemma can be proved by a standard volume argument. We include a proof for the reader's convenience.

Lemma 3.2. For $\alpha>0$ and $r \in(0,1)$ with $\alpha r<1$, there is a constant $N=N(\alpha, r)$ with the following property: If $\left\{a_{m}\right\}$ is an $r$-separated sequence, then more than $N$ of the balls $E_{\alpha r}\left(a_{m}\right)$ contain no point in common.

Proof. By (2.2), we first note that there exists a constant $C=C(n)$ such that

$$
C^{-1} \varrho^{n} \leq\left(1-|a|^{2}\right)^{-n}\left|E_{\varrho}(a)\right| \leq C\left(\frac{\varrho}{1-\varrho}\right)^{n}
$$

for all $a \in B$ and $0<\varrho<1$. Fix $z \in B$ and let $J_{z}$ be the set of all indices $m$ such that $\varrho\left(z, a_{m}\right)<\alpha r$. Let $\beta=\min \{(1-\alpha r) / 2, r\}$. Note $E_{\beta}\left(a_{m}\right) \subset$ $E_{(1+\alpha r) / 2}(z)$ for all $m \in J_{z}$. Since $E_{\beta}\left(a_{m}\right) \subset E_{r}\left(a_{m}\right)$ and the balls $E_{r}\left(a_{m}\right)$ are disjoint, it follows that

$$
\sum_{m \in J_{z}}\left|E_{\beta}\left(a_{m}\right)\right| \leq\left|E_{(1+\alpha r) / 2}(z)\right| \leq C\left(1-|z|^{2}\right)^{n}\left(\frac{1+\alpha r}{1-\alpha r}\right)^{n} .
$$

Also, for each $m \in J_{z}$, by Lemma 2.1 we have

$$
\frac{1-\alpha r}{1+\alpha r} \leq \frac{1-\left|a_{m}\right|^{2}}{1-|z|^{2}} \text {. }
$$

Thus, letting $M_{z}$ denote the cardinality of $J_{z}$, by the above we deduce

$$
\sum_{m \in J_{z}}\left|E_{\beta}\left(a_{m}\right)\right| \geq C^{-1} M_{z} \beta^{n}\left(1-|z|^{2}\right)^{n}\left(\frac{1-\alpha r}{1+\alpha r}\right)^{n} .
$$

Combining these estimates we conclude

$$
M_{z} \leq C \beta^{-n}\left(\frac{1+\alpha r}{1-\alpha r}\right)^{2 n}
$$

for some constant $C$ depending only on $n$.

Lemma 3.3. Given $0<r<\delta<1$, there exists a positive integer $N=N(\delta, r)$ with the following property: Any r-separated sequence can be decomposed into $N \delta$-separated subsequences.

Proof. Before proceeding, we mention that the hyperbolic balls and pseudohyperbolic balls are related by

$$
Q_{\tanh ^{-1} r}(a)=E_{r}(a),
$$

where $Q_{s}(a)$ denotes the hyperbolic ball with center $a$ and radius $s$. 
Now, assume $0<r<\delta<1$ and let $s=\tanh \left(2 \tanh ^{-1} \delta\right)$. By Lemma 3.2 there exists a number $N=N(r, \delta)$ such that $N$ of the balls $E_{s}\left(a_{m}\right)$ contain no point in common. Let $F_{1}$ be a maximal $\delta$-separated subcollection of $\left\{a_{m}\right\}$. Given a positive integer $n$, suppose $F_{n} \subset\left\{a_{m}\right\}$ has been chosen. If $\left\{a_{m}\right\} \backslash \bigcup_{j=1}^{n} F_{j} \neq \emptyset$, choose a maximal $\delta$-separated subcollection $F_{n+1}$ of $\left\{a_{m}\right\} \backslash \bigcup_{j=1}^{n} F_{j}$. If $\left\{a_{m}\right\}=\bigcup_{j=1}^{n} F_{j}$, then we simply let $F_{n+1}=\emptyset$. Continue this process. We claim $\left\{a_{m}\right\}=\bigcup_{j=1}^{N} F_{j}$. Suppose not. Then there exists some $a_{m_{0}} \in F_{N+1}$. For each $1 \leq j \leq N$ there exists some $a_{m_{j}} \in F_{j}$ such that $E_{\delta}\left(a_{m_{0}}\right) \cap E_{\delta}\left(a_{m_{j}}\right) \neq \emptyset$ by maximality. Now, we have $a_{m_{0}} \in \bigcap_{j=1}^{N} E_{s}\left(a_{m_{j}}\right)$ by (3.3), which is impossible.

We also need some properties of $\phi_{x}(a)$ as a function of $x$ (with $a$ fixed). So, given $a \in B$, let $\psi_{a}$ denote the function on $B$ defined by

$$
\psi_{a}(x)=\phi_{x}(a), \quad x \in B .
$$

A routine calculation shows that $\psi_{a}: B \rightarrow B$ is also invertible and

$$
\psi_{a}^{-1}(x)=\frac{1-|a|^{2}}{1-|a|^{2}|x|^{2}}\left(x+\frac{1-|x|^{2}}{1-|a|^{2}} a\right) .
$$

The Jacobian $J \psi_{a}$ of $\psi_{a}$ can be explicitly computed as follows.

Lemma 3.4. The identity

$$
J \psi_{a}(x)=\frac{1-|a|^{2}}{[x, a]^{2}}\left(\frac{1-2 a \cdot x+|a|^{2}}{[x, a]^{2}}\right)^{n-1}
$$

holds for $a, x \in B$.

Proof. Let $a, x \in B$. We have

$$
\psi_{a}(x)=x+\left(1-|x|^{2}\right) F\left(x-a^{*}\right),
$$

where $F(x)=x^{*}$. For $a=0$, we have $\psi_{0}(x)=x$ and thus $J \psi_{0}(x)=1$. So, assume $a \neq 0$. Note $F^{\prime}(x)=|x|^{-2}(I-2 Q(x))$, where $I$ is the identity matrix and $Q(x)$ is the matrix with entries $Q(x)_{i j}=x_{i} x_{j} /|x|^{2}$. Thus, denoting by $M(x, a)$ the matrix with entries $M(x, a)_{i j}=x_{j}\left(x-a^{*}\right)_{i}\left|x-a^{*}\right|^{-2}$, we have

$$
\psi_{a}^{\prime}(x)=I-2 M(x, a)+\frac{1-|x|^{2}}{\left|x-a^{*}\right|^{2}}\left[I-2 Q\left(x-a^{*}\right)\right] .
$$

Given an $n \times n$ orthogonal matrix $U$, note that

$$
U M(x, a) U^{t}=M(U x, U a) \text { and } U Q\left(x-a^{*}\right) U^{t}=Q\left(U\left(x-a^{*}\right)\right),
$$

where $U^{t}$ is the transpose of $U$. Now, choose $U$ such that $U\left(x-a^{*}\right)=$ $\left(\left|x-a^{*}\right|, 0, \ldots, 0\right)$. Note that the first entry of $Q\left(U\left(x-a^{*}\right)\right)$ is 1 and all others are 0 . Also, row vectors of $M(U x, U a)$ except the first one are all zero. Thus $U\left[\left(\psi_{a}^{-1}\right)^{\prime}(x)\right] U^{t}$ is an upper triangular matrix. Since the first 
entry of $M(U x, U a)$ is

$$
\frac{U x \cdot\left[U x-(U a)^{*}\right]}{\left|U x-(U a)^{*}\right|^{2}}=\frac{U x \cdot U\left(x-a^{*}\right)}{\left|U\left(x-a^{*}\right)\right|^{2}}=\frac{x \cdot\left(x-a^{*}\right)}{\left|x-a^{*}\right|^{2}},
$$

we see from (3.4) that the first entry of $U\left[\left(\psi_{a}^{-1}\right)^{\prime}(x)\right] U^{t}$ is

$$
1-2 \frac{x \cdot\left(x-a^{*}\right)}{\left|x-a^{*}\right|^{2}}-\frac{1-|x|^{2}}{\left|x-a^{*}\right|^{2}}=\frac{1-|a|^{2}}{[x, a]^{2}}
$$

and the other diagonal entries are

$$
1+\frac{1-|x|^{2}}{\left|x-a^{*}\right|^{2}}=1+\frac{|a|^{2}\left(1-|x|^{2}\right)}{[x, a]^{2}}=\frac{1-2 a \cdot x+|a|^{2}}{[x, a]^{2}} .
$$

Accordingly, we conclude

$$
J \psi_{a}(x)=\operatorname{det} U\left[\left(\psi_{a}^{-1}\right)^{\prime}(x)\right] U^{t}=\frac{1-|a|^{2}}{[x, a]^{2}}\left(\frac{1-2 a \cdot x+|a|^{2}}{[x, a]^{2}}\right)^{n-1},
$$

as required.

As a consequence of Lemma 3.4, we have

$$
\left(1-|x|^{2}\right)^{\alpha}=\left(1-\left|\psi_{a}(x)\right|^{2}\right)^{\alpha} J \psi_{a}(x)\left(\frac{[x, a]^{2}}{1-|a|^{2}}\right)^{\alpha+1}\left(\frac{[x, a]^{2}}{1-2 a \cdot x+|a|^{2}}\right)^{n-1}
$$

for $a, x \in B$ and $\alpha$ real. Thus, making a change of variables, we have the following inequality.

Corollary 3.5. Given $\alpha$ real and a compact subset $K \subset B$, there is a constant $C=C(K, \alpha)$ such that

$$
\int_{B} f\left(\phi_{x}(a)\right)\left(1-|x|^{2}\right)^{\alpha} d x \leq C \int_{B} f(x)\left(1-|x|^{2}\right)^{\alpha} d x
$$

for $a \in K$ and measurable functions $f \geq 0$ on $B$.

Now, we prove that the $L_{\alpha}^{p}$-behavior of the averaging functions of positive measures is independent of the radii. In what follows, $L_{0}$ denotes the space of all functions $f$ bounded on $B$ and such the $f(x) \rightarrow 0$ as $|x| \rightarrow 1$.

Proposition 3.6. Let $0<p \leq \infty, r, \delta \in(0,1)$ and $\alpha$ be real. Assume $\mu \geq 0$. Then the following statements hold:

(a) $\widehat{\mu}_{r} \in L_{\alpha}^{p}$ if and only if $\widehat{\mu}_{\delta} \in L_{\alpha}^{p}$.

(b) $\widehat{\mu}_{r} \in L_{0}$ if and only if $\widehat{\mu}_{\delta} \in L_{0}$.

Proof. We first prove (a). By symmetry it suffices to establish the estimate

$$
\left\|\widehat{\mu}_{r}\right\|_{L_{\alpha}^{p}} \lesssim\left\|\widehat{\mu}_{\delta}\right\|_{L_{\alpha}^{p}}
$$

Since the case $r \leq \delta$ is easily treated by (2.5), we may assume $\delta<r$. Choose a finite set $\left\{a_{1}, \ldots, a_{N}\right\}$ in $E_{r}(0)$ which is maximal subject to the condition 
$E_{\delta / 2}\left(a_{i}\right) \cap E_{\delta / 2}\left(a_{j}\right)=\emptyset$ for $i \neq j$. We then have $E_{r}(0) \subset \bigcup_{j=1}^{N} E_{\delta}\left(a_{j}\right)$ by maximality and hence

$$
E_{r}(x)=\phi_{x} E_{r}(0) \subset \bigcup_{j=1}^{N} \phi_{x} E_{\delta}\left(a_{j}\right)=\bigcup_{j=1}^{N} E_{\delta}\left(\phi_{x}\left(a_{j}\right)\right)
$$

for every $x \in B$. Since $\phi_{x}\left(a_{j}\right) \in E_{r}(x)$, we note by $(2.5)$ that $\left|E_{\delta}\left(\phi_{x}\left(a_{j}\right)\right)\right|$ $\approx\left|E_{r}(x)\right|$ for all $j$ and $x \in B$. Consequently,

$$
\widehat{\mu}_{r}(x) \leq \sum_{j=1}^{N} \frac{\mu\left[E_{\delta}\left(\phi_{x}\left(a_{j}\right)\right)\right]}{\left|E_{r}(x)\right|}=\sum_{j=1}^{N} \frac{\left|E_{\delta}\left(\phi_{x}\left(a_{j}\right)\right)\right|}{\left|E_{r}(x)\right|} \widehat{\mu}_{\delta}\left(\phi_{x}\left(a_{j}\right)\right) \approx \sum_{j=1}^{N} \widehat{\mu}_{\delta}\left(\phi_{x}\left(a_{j}\right)\right)
$$

for every $x \in B$. This implies (3.5) for $p=\infty$. For $p<\infty$, the above yields

$$
\widehat{\mu}_{r}(x)^{p} \lesssim \max \left\{1, N^{p-1}\right\} \sum_{j=1}^{N} \widehat{\mu}_{\delta}\left(\phi_{x}\left(a_{j}\right)\right)^{p}
$$

for all $x \in B$. Now, integrating both sides of (3.6) against the measure $d V_{\alpha}(x)$, we deduce (3.5) by Corollary 3.5.

Now, we prove (b). It is easily seen that $\left|\phi_{x}\right| \rightarrow 1$ as $|x| \rightarrow 1$ uniformly on every compact subset of $B$. So, we see that $\widehat{\mu}_{\delta} \in L_{0}$ implies $\widehat{\mu}_{r} \in L_{0}$ by (3.6), and vice versa by symmetry.

The next two lemmas will be used in establishing the discretized version of Proposition 3.6. The first one is a special case of [5, Theorem 3.6].

Lemma 3.7. Let $1 \leq p \leq \infty$ and $\alpha$ be real. Then the Berezin transform is bounded on $L_{\alpha}^{p}$ if and only if $-n<(\alpha+1) / p<1$.

Lemma 3.8. Given $\delta \in(0,1)$, there exists a constant $C_{\delta}$ such that $\widetilde{\mu} \leq$ $C_{\delta} \widetilde{\widehat{\mu}}_{\delta}$ for $\mu \geq 0$.

In the proof below and elsewhere, $\chi_{E}$ denotes the characteristic function of $E \subset B$.

Proof. Fix $\delta \in(0,1)$. We will actually prove a more general fact asserting that

$$
\int_{B} f d \mu \leq C_{\delta} \int_{B} f \widehat{\mu}_{\delta} d V
$$

for all $f \geq 0$ subharmonic on $B$ and $\mu \geq 0$.

Let $\mu \geq 0$ and $f$ be a positive subharmonic function. By (2.2) and subharmonicity we have

$$
f(x) \lesssim \frac{1}{\left|E_{\delta}(x)\right|} \int_{E_{\delta}(x)} f(y) d y
$$


for all $x \in B$. Also, by Lemma 2.1,

$$
\int_{E_{\delta}(y)} \frac{d \mu(x)}{\left|E_{\delta}(x)\right|} \approx \int_{E_{\delta}(y)} \frac{d \mu(x)}{\left(1-|x|^{2}\right)^{n}} \approx \frac{\mu\left(E_{\delta}(y)\right)}{\left(1-|y|^{2}\right)^{n}} \approx \widehat{\mu}_{\delta}(y)
$$

for $y \in B$. The constants suppressed above depend only on $\delta$ (and $n$ ). Now, one may use the equality $\chi_{E_{\delta}(x)}(y)=\chi_{E_{\delta}(y)}(x)$ and Fubini's theorem to conclude the proof.

Now, we are ready to prove the main result of this section.

TheOrem 3.9. Let $0<p \leq \infty, r, \delta \in(0,1)$ and $\alpha$ be real. Let $\mu \geq 0$ and $\mathfrak{a}=\left\{a_{m}\right\}$ be an $r$-lattice. Then the following two statements are equivalent:

(a) $\widehat{\mu}_{\delta} \in L_{\alpha}^{p}$.

(b) $\left\{\widehat{\mu}_{r}\left(a_{m}\right)\right\} \in \ell^{p, n+\alpha}(\mathfrak{a})$.

Moreover, if

$$
\max \left\{1+\alpha, 1+\frac{\alpha}{n},-\frac{\alpha+1}{n}\right\}<p \leq \infty,
$$

then the above statements are also equivalent to

(c) $\widetilde{\mu} \in L_{\alpha}^{p}$.

It is clear from (3.1) that (b) is a discretized version of (a). Thus the equivalence of (a) and (b) is not surprising. However, it seems quite interesting to see that the restricted range (3.8) is best possible for (c); see Section 5.

Proof. $(\mathrm{a}) \Rightarrow(\mathrm{b})$. Assume (a). Pick a positive $\varepsilon<\min \{r / 2,1-r\}$. By Proposition 3.6(a), in order to prove (b), it is sufficient to establish the estimate

$$
\left\|\left\{\widehat{\mu}_{r}\left(a_{m}\right)\right\}\right\|_{\ell^{p, n+\alpha}(\mathfrak{a})} \lesssim\left\|\widehat{\mu}_{r+\varepsilon}\right\|_{L_{\alpha}^{p}} .
$$

Since $\varepsilon<r / 2$ and $\left\{a_{m}\right\}$ is an $r$-lattice, the balls $E_{\varepsilon}\left(a_{m}\right)$ are pairwise disjoint. Also, given $x \in E_{\varepsilon}\left(a_{m}\right)$, we have $E_{r}\left(a_{m}\right) \subset E_{r+\varepsilon}(x)$ and thus

$$
\widehat{\mu}_{r+\varepsilon}(x) \geq \frac{\mu\left[E_{r}\left(a_{m}\right)\right]}{\left|E_{r+\varepsilon}(x)\right|}=\frac{\left|E_{r}\left(a_{m}\right)\right|}{\left|E_{r+\varepsilon}(x)\right|} \widehat{\mu}_{r}\left(a_{m}\right) \approx \widehat{\mu}_{r}\left(a_{m}\right)
$$

by (2.5). This implies (3.9) for $p=\infty$. For $p<\infty$, it follows from (3.1) that $\int_{B} \widehat{\mu}_{r+\varepsilon}(x)^{p} d V_{\alpha}(x) \gtrsim \sum_{m} \int_{E_{\varepsilon}\left(a_{m}\right)} \widehat{\mu}_{r+\varepsilon}(x)^{p} d V_{\alpha}(x) \gtrsim \sum_{m} \widehat{\mu}_{r}\left(a_{m}\right)^{p}\left(1-\left|a_{m}\right|^{2}\right)^{n+\alpha}$ and thus (3.9) holds.

$(\mathrm{b}) \Rightarrow(\mathrm{a})$. Assume (b). In order to prove (a), we may assume $\delta<1-r$ by Proposition 3.6(a). Given $x \in B$, let $N(x)=\left\{m: E_{r}\left(a_{m}\right) \cap E_{\delta}(x) \neq \emptyset\right\}$. Since $\left\{a_{m}\right\}$ is an $r$-lattice, we have $E_{\delta}(x) \subset \bigcup_{m \in N(x)} E_{r}\left(a_{m}\right)$ and thus 
$\mu\left[E_{\delta}(x)\right] \leq \sum_{m \in N(x)} \mu\left[E_{r}\left(a_{m}\right)\right]$. It follows from this and (2.5) that

$$
\widehat{\mu}_{\delta}(x) \lesssim \sum_{m \in N(x)} \frac{\left|E_{r}\left(a_{m}\right)\right|}{\left|E_{\delta}(x)\right|} \widehat{\mu}_{r}\left(a_{m}\right) \approx \sum_{m \in N(x)} \widehat{\mu}_{r}\left(a_{m}\right) .
$$

Meanwhile, for $m \in N(x)$, since the balls $E_{\varepsilon}\left(a_{m}\right)$ are pairwise disjoint and are all contained in $E_{r+\delta+\varepsilon}(x)$ for a fixed positive number $\varepsilon<$ $\min \{r / 2,1-r-\delta\}$, we see from $(2.5)$ that

$$
N=N(r, \delta, \varepsilon):=\sup _{x \in B} \sharp N(x)<\infty,
$$

where $\sharp N(x)$ denotes the number of elements in the set $N(x)$. Thus, we have $\left\|\widehat{\mu}_{\delta}\right\|_{L^{\infty}(\lambda)} \lesssim\left\|\left\{\widehat{\mu}_{r}\left(a_{m}\right)\right\}\right\|_{L^{\infty}(\lambda)}$ by (3.10). Also, for $p<\infty$, by (3.10) again,

$$
\widehat{\mu}_{\delta}(x)^{p} \lesssim \max \left\{1, N^{p-1}\right\} \sum_{m \in N(x)} \widehat{\mu}_{r}\left(a_{m}\right)^{p}
$$

for all $x \in B$. Now, integrating both sides of the above against the measure $d V_{\alpha}(x)$ and then applying Fubini's theorem, we obtain

$$
\int_{B} \widehat{\mu}_{\delta}(x)^{p} d V_{\alpha}(x) \lesssim \int_{B} \sum_{m \in N(x)} \widehat{\mu}_{r}\left(a_{m}\right)^{p} d V_{\alpha}(x)=\sum_{m} \widehat{\mu}_{r}\left(a_{m}\right)^{p} V_{\alpha}[Q(m)],
$$

where $Q(m)=\left\{x \in B: E_{r}\left(a_{m}\right) \cap E_{\delta}(x) \neq \emptyset\right\}$. Note that $Q(m) \subset E_{r+\delta}\left(a_{m}\right)$ and thus $V_{\alpha}[Q(m)] \leq V_{\alpha}\left[E_{r+\delta}\left(a_{m}\right)\right] \approx\left(1-\left|a_{m}\right|^{2}\right)^{n+\alpha}$ by (3.1). Combining this with the above estimate, we conclude that (a) holds.

$(\mathrm{c}) \Rightarrow(\mathrm{a})$. This follows from Lemma 3.1 and Proposition 3.6(a).

Now, we assume (3.8) and prove that either (a) or (b) implies (c). If $p \geq 1$, one may use Lemmas 3.7 and 3.8 to see that (a) implies (c). If $p<1$, we have $\alpha<0$ by (3.8) and thus (3.8) reduces to

$$
\max \left\{1+\frac{\alpha}{n},-\frac{\alpha+1}{n}\right\}<p<1
$$

We now assume this and proceed to prove that (b) implies (c).

(b) $\Rightarrow(\mathrm{c})$. Assume $\left\{\widehat{\mu}_{r}\left(a_{m}\right)\right\} \in \ell^{p, n+\alpha}(\mathfrak{a})$. By (2.6) and Lemma 2.2, we note

$$
\begin{aligned}
\widetilde{\mu}(x) & =\left(1-|x|^{2}\right)^{n} \int_{B}|R(x, y)|^{2} d \mu(y) \leq\left(1-|x|^{2}\right)^{n} \sum_{m} \int_{E_{r}\left(a_{m}\right)} \frac{1}{[x, y]^{2 n}} d \mu(y) \\
& \lesssim \sum_{m} \mu\left[E_{r}\left(a_{m}\right)\right] \frac{\left(1-|x|^{2}\right)^{n}}{\left[x, a_{m}\right]^{2 n}} \approx \sum_{m}\left(1-\left|a_{m}\right|^{2}\right)^{n} \widehat{\mu}_{r}\left(a_{m}\right) \frac{\left(1-|x|^{2}\right)^{n}}{\left[x, a_{m}\right]^{2 n}}
\end{aligned}
$$

and thus (recall $p<1$ )

$$
\widetilde{\mu}(x)^{p} \lesssim \sum_{m}\left(1-\left|a_{m}\right|^{2}\right)^{n p} \widehat{\mu}_{r}\left(a_{m}\right)^{p} \frac{\left(1-|x|^{2}\right)^{n p}}{\left[x, a_{m}\right]^{2 n p}}
$$


for all $x \in B$. Now, since $n p+\alpha>-1$ and $n p-n-\alpha>0$ by (3.11), from Lemma 2.5 we obtain

$$
\begin{aligned}
\int_{B} \widetilde{\mu}(x)^{p} d V_{\alpha}(x) & \lesssim \sum_{m} \widehat{\mu}_{r}\left(a_{m}\right)^{p}\left(1-\left|a_{m}\right|^{2}\right)^{n p} \int_{B} \frac{\left(1-|x|^{2}\right)^{n p+\alpha}}{\left[x, a_{m}\right]^{2 n p}} d x \\
& \approx \sum_{m} \widehat{\mu}_{r}\left(a_{m}\right)^{p}\left(1-\left|a_{m}\right|^{2}\right)^{n+\alpha}
\end{aligned}
$$

and thus (c) holds.

We also have the little oh version of Theorem 3.9. We let $\ell_{0}$ denote the space of all complex sequences vanishing at $\infty$.

Proposition 3.10. Let $r, \delta \in(0,1)$ and $\mu \geq 0$. Suppose $\mathfrak{a}=\left\{a_{m}\right\}$ is an $r$-lattice. Then the following statements are equivalent:

(a) $\widehat{\mu}_{\delta} \in L_{0}$.

(b) $\left\{\widehat{\mu}_{r}\left(a_{m}\right)\right\} \in \ell_{0}$.

(c) $\widetilde{\mu} \in L_{0}$.

Proof. Since $\lim _{m \rightarrow \infty}\left|a_{m}\right|=1$, we have (a) $\Rightarrow(\mathrm{b})$. Also, (c) $\Rightarrow$ (a) from Lemma 3.1 and Proposition 3.6(b). Now, we prove (b) $\Rightarrow(\mathrm{c})$. Note $1-|a| \leq$ $[x, a]$ for all $x, a \in B$. Given a positive integer $j$, put $M_{j}=\sup _{m>j} \widehat{\mu}_{r}\left(a_{m}\right)$ and let $N$ be the positive integer provided by Lemma 3.2. By the proof of $(\mathrm{b}) \Rightarrow(\mathrm{c})$ of Theorem 3.9 and by Lemmas 2.2 and 2.5 , for each $j$ we have

$$
\begin{aligned}
\widetilde{\mu}(x) & \lesssim \sum_{m} \widehat{\mu}_{r}\left(a_{m}\right) \frac{\left(1-|x|^{2}\right)^{n}\left(1-\left|a_{m}\right|^{2}\right)^{n}}{\left[x, a_{m}\right]^{2 n}} \\
& \lesssim \sum_{m<j} \widehat{\mu}_{r}\left(a_{m}\right) \frac{\left(1-|x|^{2}\right)^{n}}{\left(1-\left|a_{m}\right|^{2}\right)^{n}}+M_{j} \sum_{m \geq j} \int_{E_{r}\left(a_{m}\right)} \frac{\left(1-|x|^{2}\right)^{n}}{[x, y]^{2 n}} d y \\
& \lesssim \sum_{m<j} \widehat{\mu}_{r}\left(a_{m}\right) \frac{\left(1-|x|^{2}\right)^{n}}{\left(1-\left|a_{m}\right|^{2}\right)^{n}}+N M_{j}\left(1-|x|^{2}\right)^{n} \int_{B} \frac{d y}{[x, y]^{2 n}} \\
& \lesssim \sum_{m<j} \widehat{\mu}_{r}\left(a_{m}\right) \frac{\left(1-|x|^{2}\right)^{n}}{\left(1-\left|a_{m}\right|^{2}\right)^{n}}+N M_{j} .
\end{aligned}
$$

Thus, fixing $j$ and taking the limit $|x| \rightarrow 1$ in the above, we obtain

$$
\lim _{|x| \rightarrow 1} \widetilde{\mu}(x) \lesssim \sup _{m \geq j} \widehat{\mu}_{r}\left(a_{m}\right)
$$

for each $j$. Now, letting $j \rightarrow \infty$, we get (c) by assumption.

As a consequence of Theorem 3.9 and Proposition 3.10, we have the following. 
Corollary 3.11. Let $0<p \leq \infty, r, \delta \in(0,1)$ and $\alpha$ be real. Assume $\mu \geq 0$. Then $\left\{\widehat{\mu}_{r}\left(a_{m}\right)\right\} \in \ell^{p, \alpha}(\mathfrak{a})$ (resp. $\left.\ell_{0}\right)$ for all $r$-lattices $\mathfrak{a}=\left\{a_{m}\right\}$ if and only if $\left\{\widehat{\mu}_{\delta}\left(a_{m}\right)\right\} \in \ell^{p, \alpha}(\mathfrak{a})$ (resp. $\left.\ell_{0}\right)$ for some $\delta$-lattice $\mathfrak{a}=\left\{a_{m}\right\}$.

4. Schatten class Toeplitz operators. In this section we prove Theorem 1.1. We first briefly review the notion of Schatten class operators. For a positive compact operator $T$ on a separable Hilbert space $H$, there exist an orthonormal set $\left\{e_{m}\right\}$ in $H$ and a sequence $\left\{\lambda_{m}\right\}$ that decreases to 0 such that

$$
T x=\sum_{m} \lambda_{m}\left\langle x, e_{m}\right\rangle e_{m}
$$

for all $x \in H$, where $\langle$,$\rangle denotes the inner product on H$. For $0<p<\infty$, we say that a positive operator $T$ belongs to the Schatten p-class $S_{p}(H)$ if

$$
\|T\|_{p}:=\left\{\sum_{m} \lambda_{m}^{p}\right\}^{1 / p}<\infty .
$$

More generally, given a compact operator $T$ on $H$, we say that $T \in S_{p}(H)$ if the positive operator $|T|=\left(T^{*} T\right)^{1 / 2}$ belongs to $S_{p}(H)$, and we define $\|T\|_{p}=\||T|\|_{p}$. Of course, we will take $H=b^{2}$ in our applications below and, in that case, we put $S_{p}=S_{p}\left(b^{2}\right)$.

First, we recall a couple of basic facts about $S_{p}(H)$, which we need later. The following lemma is taken from $[9$, Lemma 5] where further references are given.

Lemma 4.1. Let $0<p \leq 2$ and $T$ be a compact operator on $H$. Then

$$
\|T\|_{p}^{p} \leq \sum_{i} \sum_{j}\left|\left\langle T e_{i}, e_{j}\right\rangle\right|^{p}
$$

for any orthonormal basis $\left\{e_{k}\right\}$ of $H$.

The proof of the following lemma is implicit in that of [14, Theorem 1.4.7], where the equality for the case $1 \leq p<\infty$ is proved.

Lemma 4.2. Let $0<p<1$ and $T$ be a positive compact operator on $H$. Then

$$
\|T\|_{p}^{p} \leq \sup \sum_{m}\left\langle T e_{m}, e_{m}\right\rangle^{p},
$$

where sup is taken over all orthonormal bases $\left\{e_{m}\right\}$ of $H$.

For $0<q \leq \infty$, we use the notation $\ell^{q}$ for the $q$-summable sequence space. Let $\left\{a_{m}\right\}$ be a sequence in $B$ and $k \geq 0$ be an integer. For $\xi=$ $\left\{\xi_{m}\right\} \in \ell^{2}$, let $Q_{k}(\xi)$ denote the formal series defined by

$$
Q_{k}(\xi)(x)=\sum_{m} \xi_{m}\left(1-\left|a_{m}\right|^{2}\right)^{k+n / 2} R_{k}\left(x, a_{m}\right), \quad x \in B .
$$


For the proof of Theorem 1.1, we need the following representation theorem for $b^{2}$-harmonic functions, which is essentially proved in [8, Theorem 3]. In [8] Coifman and Rochberg used local estimates directly instead of Möbius transformations and provided only a sketch of proof. We refer to [6] for a detailed proof via Möbius transformations.

Lemma 4.3. Let $k \geq 0$ be an integer. Then the following statements hold:

(a) If $\left\{a_{m}\right\}$ is a separated sequence, then $Q_{k}: \ell^{2} \rightarrow b^{2}$ is bounded.

(b) There exists $\delta_{k}>0$ with the following property: If $\left\{a_{m}\right\}$ is a $\delta$-lattice with $\delta<\delta_{k}$, then $Q_{k}: \ell^{2} \rightarrow b^{2}$ is onto.

We also need a lemma which is useful in estimating certain quantities related to the operators $Q_{k}$.

LEMma 4.4. Let $s, t$ be real and $r, \delta \in(0,1)$. Then

$$
\frac{\left(1-|b|^{2}\right)^{t}}{[x, b]^{s}} \approx \int_{E_{r}(b)} \frac{\left(1-|y|^{2}\right)^{t}}{[a, y]^{s}} d \lambda(y)
$$

whenever $b \in B$ and $x \in E_{\delta}(a)$. The constants suppressed above depend only on $n, s, t, r$ and $\delta$.

Proof. Let $a, b \in B$. By Lemma 2.2, we note $[x, b]^{s} \approx[a, b]^{s}$ for $x \in E_{\delta}(a)$. Also, by Lemmas 2.1 and 2.2,

$$
\frac{\left(1-|b|^{2}\right)^{t}}{[a, b]^{s}} \approx \frac{\left(1-|y|^{2}\right)^{t}}{[a, y]^{s}}
$$

for $y \in E_{r}(b)$. Thus we conclude the proof by (3.1). One can check that all the constants suppressed above depend on parameters, but not on particular points.

We are now ready to prove the following version of Theorem 1.1.

Theorem 4.5. Let $0<p<\infty, \mu \geq 0$ and assume that $\left\{a_{m}\right\}$ is an $r$-lattice. Then the following three statements are equivalent:

(a) $T_{\mu} \in S_{p}$.

(b) $\left\{\widehat{\mu}_{r}\left(a_{m}\right)\right\} \in \ell^{p}$.

(c) $\widehat{\mu}_{r} \in L^{p}(\lambda)$.

Moreover, if $(n-1) / n<p$, then the above statements are also equivalent to

(d) $\widetilde{\mu} \in L^{p}(\lambda)$.

For $p \geq 1$, we have $T_{\mu} \in S_{p}$ if and only if $\widetilde{\mu} \in L^{p}(\lambda)$ by [7, Theorem 3.13]. So, by Theorem 3.9 (with $\alpha=-n$ ), we only need to prove that (a) and (b) are equivalent for $0<p<1$. 
Proof of $(\mathrm{a}) \Rightarrow(\mathrm{b})$. Assume (a). By Corollary 3.11, it suffices to consider $r$ sufficiently small. Fix a large integer $k>0$ such that

$$
k>\frac{n(2-p)}{2 p}
$$

and let $r_{k}$ be the constant provided by Lemma 2.3. To begin with, fix $r<$ $\min \left\{r_{k}, 1 / 2\right\}$ and let $2 r<\delta<1$. Later the constant $\delta$ will be chosen to be sufficiently close to 1 .

By Lemma 3.3, we can decompose the lattice $\left\{a_{m}\right\}$ into finitely many, say $N, \delta / 2$-separated subsequences. Let $\left\{b_{m}\right\}$ be one of such subsequences and define a measure $\nu$ by

$$
d \nu=\sum_{m} \chi_{E_{r}\left(b_{m}\right)} d \mu .
$$

Note that the balls $E_{r}\left(b_{m}\right)$ are pairwise disjoint, because $2 r<\delta$. Also, note that $\left\|T_{\nu}\right\|_{p} \leq\left\|T_{\mu}\right\|_{p}$, because $0 \leq \nu \leq \mu$.

Now, we introduce some auxiliary operators. Fix an orthonormal basis $\left\{e_{m}\right\}$ for $b^{2}$ and let $J: b^{2} \rightarrow \ell^{2}$ be the unitary operator defined by $J f=$ $\left\{\left\langle f, e_{m}\right\rangle\right\}$ for $f \in b^{2}$. Put $A=Q_{k} J$, where $Q_{k}$ is the operator associated with the sequence $\left\{b_{m}\right\}$ as in (4.1). By Lemma 4.3(a), $A: b^{2} \rightarrow b^{2}$ is bounded. Put

$$
h_{m}:=A e_{m}=\left(1-\left|b_{m}\right|^{2}\right)^{k+n / 2} R_{k}\left(\cdot, b_{m}\right)
$$

for simplicity. Put $T=A^{*} T_{\nu} A$. Note $T \geq 0$. Since $A$ is bounded and $T_{\nu} \in$ $S_{p}$, we have $T \in S_{p}$ with $\|T\|_{p} \leq\|A\|^{2}\left\|T_{\nu}\right\|_{p} \leq\|A\|^{2}\left\|T_{\mu}\right\|_{p}$ (see, for example, [10]).

Note that

$$
\left\langle T e_{j}, e_{m}\right\rangle=\left\langle T_{\nu} h_{j}, h_{m}\right\rangle=\int_{B} h_{j} h_{m} d \nu
$$

for each $m$ and $j$. The change of the order of integrations, which is implicit in the last equality above, is justified by [7, Lemma 3.8]. Since $\nu=\mu$ on the balls $E_{r}\left(b_{m}\right)$, we see from Lemma 3.1 that

$$
\sum_{m}\left\langle T e_{m}, e_{m}\right\rangle^{p} \geq C_{1} \sum_{m} \widehat{\mu}_{r}\left(b_{m}\right)^{p}
$$

for some constant $C_{1}=C_{1}(n, p, k, r)>0$. We claim that there exists a constant $C_{\delta}=C_{\delta}(n, p, k, r)>0$ such that

$$
\sum_{m \neq j}\left|\left\langle T e_{j}, e_{m}\right\rangle\right|^{p} \leq C_{\delta} \sum_{m} \widehat{\mu}_{r}\left(b_{m}\right)^{p}
$$

and $C_{\delta} \rightarrow 0$ as $\delta \rightarrow 1$.

Now, we prove (4.4). Let $m$ and $j$ be given. Since

$$
\left\langle T e_{j}, e_{m}\right\rangle=\sum_{i} \int_{E_{r}\left(b_{i}\right)} h_{j} h_{m} d \mu
$$


we have

$$
\left|\left\langle T e_{j}, e_{m}\right\rangle\right|^{p} \leq \sum_{i}\left\{\int_{E_{r}\left(b_{i}\right)}\left|h_{j} h_{m}\right| d \mu\right\}^{p} .
$$

Note that by (2.6) and Lemma 4.4 we have

$$
\left|h_{j}(x)\right|^{p} \lesssim\left\{\frac{\left(1-\left|b_{j}\right|^{2}\right)^{n / 2+k}}{\left[x, b_{j}\right]^{n+k}}\right\}^{p} \approx I_{i j}, \quad x \in E_{r}\left(b_{i}\right),
$$

for all $i, j$, where

$$
I_{i j}=\int_{E_{r}\left(b_{j}\right)}\left\{\frac{\left(1-|y|^{2}\right)^{n / 2+k}}{\left[b_{i}, y\right]^{n+k}}\right\}^{p} d \lambda(y) .
$$

It follows that

$$
\left\{\int_{E_{r}\left(b_{i}\right)}\left|h_{j} h_{m}\right| d \mu\right\}^{p} \lesssim \mu\left[E_{r}\left(b_{i}\right)\right]^{p} I_{i j} I_{i m} \approx\left(1-\left|b_{i}\right|^{2}\right)^{n p} \widehat{\mu}_{r}\left(b_{i}\right)^{p} I_{i j} I_{i m}
$$

for all $i, j$ and $m$. Thus, by (4.5), we have

$$
\left|\left\langle T e_{j}, e_{m}\right\rangle\right|^{p} \lesssim \sum_{i}\left(1-\left|b_{i}\right|^{2}\right)^{n p} \widehat{\mu}_{r}\left(b_{i}\right)^{p} I_{i j} I_{i m}
$$

Now, summing up both sides of the above over all $m$ and $j$ with $m \neq j$ and then changing the order of summations, we obtain

$$
\sum_{m \neq j}\left|\left\langle T e_{j}, e_{m}\right\rangle\right|^{p} \lesssim \sum_{i}\left(1-\left|b_{i}\right|^{2}\right)^{p n} \widehat{\mu}_{r}\left(b_{i}\right)^{p} I_{i}
$$

where $I_{i}=\sum_{m \neq j} I_{i j} I_{i m}$. Since the balls $E_{r}\left(b_{m}\right)$ are pairwise disjoint, we have

$$
\begin{aligned}
I_{i} & =\sum_{m \neq j} \int_{E_{r}\left(b_{j}\right)} \int_{E_{r}\left(b_{m}\right)}\left\{\frac{\left(1-|x|^{2}\right)^{n / 2+k}}{\left[x, b_{i}\right]^{n+k}} \frac{\left(1-|y|^{2}\right)^{n / 2+k}}{\left[y, b_{i}\right]^{n+k}}\right\}^{p} d \lambda(x) d \lambda(y) \\
& =\iint_{\Omega_{r, \delta}}\left\{\frac{\left(1-|x|^{2}\right)^{n / 2+k}}{\left[x, b_{i}\right]^{n+k}} \frac{\left(1-|y|^{2}\right)^{n / 2+k}}{\left[y, b_{i}\right]^{n+k}}\right\}^{p} d \lambda(x) d \lambda(y),
\end{aligned}
$$

where

$$
\Omega_{r, \delta}=\bigcup_{j \neq m} E_{r}\left(b_{j}\right) \times E_{r}\left(b_{m}\right) .
$$

Note that, for $(x, y) \in \Omega_{r, \delta}$, we have

$$
\beta(x, y) \geq \frac{1}{2} \log \frac{1+\delta}{1-\delta}-\log \frac{1+r}{1-r}
$$

recall that $\beta$ denotes the hyperbolic distance given by (2.1). Thus, denoting by $G_{r, \delta}$ the set of all $(z, w)$ satisfying (4.7), we note that the region $G_{r, \delta}$ is Möbius invariant. Also, note that the measure $d \lambda$ is Möbius invariant. Thus, 
making the change of variables $x=\phi_{b_{i}}(z)$ and $y=\phi_{b_{i}}(w)$, we have

$$
\begin{aligned}
I_{i} & \lesssim \iint_{G_{r, \delta}}\left\{\frac{\left(1-|x|^{2}\right)^{n / 2+k}}{\left[x, b_{i}\right]^{n+k}} \frac{\left(1-|y|^{2}\right)^{n / 2+k}}{\left[y, b_{i}\right]^{n+k}}\right\}^{p} d \lambda(x) d \lambda(y) \\
& =\frac{1}{\left(1-\left|b_{i}\right|^{2}\right)^{p n}} \iint_{G_{r, \delta}}\left\{\frac{\left(1-|z|^{2}\right)^{n / 2+k}}{\left[z, b_{i}\right]^{k}} \frac{\left(1-|w|^{2}\right)^{n / 2+k}}{\left[w, b_{i}\right]^{k}}\right\}^{p} d \lambda(z) d \lambda(w) .
\end{aligned}
$$

Since $0<p<1$, we note $1<2 /(2-p)$. Choose $t$ such that $1<t<2 /(2-p)$ and let $s$ be the conjugate exponent of $t$. Then, by Hölder's inequality, we see that

$$
\begin{gathered}
\iint_{G_{r, \delta}}\left\{\frac{\left(1-|z|^{2}\right)^{n / 2+k}}{\left[z, b_{i}\right]^{k}} \frac{\left(1-|w|^{2}\right)^{n / 2+k}}{\left[w, b_{i}\right]^{k}}\right\}^{p} d \lambda(z) d \lambda(w) \\
=\iint_{G_{r, \delta}} \frac{\left(1-|z|^{2}\right)^{p(n / 2+k)-n}}{\left[z, b_{i}\right]^{p k}} \frac{\left(1-|w|^{2}\right)^{p(n / 2+k)-n}}{\left[w, b_{i}\right]^{p k}} d z d w \\
\leq\left|G_{r, \delta}\right|^{1 / s}\left\{\sup _{i} \int_{B} \frac{\left(1-|z|^{2}\right)^{p t(n / 2+k)-n t}}{\left[z, b_{i}\right]^{p t k}} d z\right\}^{2 / t},
\end{gathered}
$$

where $\left|G_{r, \delta}\right|$ denotes the $2 n$-dimensional volume of $G_{r, \delta} \subset B \times B$. Note that $p t(n / 2+k)-n t>-1$ and $n t-p t n / 2<n$ by our choice of $k$ and $t$. Thus the supremum in the braces above is finite by Lemma 2.5. Hence, combining these observations, we have

$$
I_{i} \leq C \frac{\left|G_{r, \delta}\right|^{1 / s}}{\left(1-\left|b_{i}\right|^{2}\right)^{p n}}
$$

for some constant $C=C(n, p, k)$ independent of $i$. Note $\left|G_{r, \delta}\right| \rightarrow 0$ as $\delta \rightarrow 1$ by (4.7). Finally, fixing $\delta$ sufficiently close to 1 , we see from (4.6) that $(4.4)$ holds.

We introduce some auxiliary operators. Associated with $T$ is the diagonal operator $D: b^{2} \rightarrow b^{2}$ whose diagonal components are inherited from $T$. More precisely, $D$ is defined by

$$
D f=\sum_{m}\left\langle T e_{m}, e_{m}\right\rangle\left\langle f, e_{m}\right\rangle e_{m}
$$

for $f \in b^{2}$. Note that $D$ is compact by [14, Proposition 1.3.10] and positive. Put $E=T-D$. Note that

$$
\left\langle D e_{m}, e_{m}\right\rangle=\left\langle T e_{m}, e_{m}\right\rangle \text { and }\left\langle E e_{j}, e_{m}\right\rangle=\left\langle T e_{j}, e_{m}\right\rangle
$$

for any $m$ and $j$ with $m \neq j$.

Assume for the moment that $\mu$ is supported on a compact set. Then a standard volume argument as in the proof of Theorem 3.9 shows that only 
finitely many of the balls $E_{r}\left(a_{m}\right)$ can intersect the support of $\mu$. Thus it follows from (4.4), (4.8) and Lemma 4.1 that $E \in S_{p}$ with

$$
\|E\|_{p}^{p} \leq \sum_{m \neq j}\left|\left\langle E e_{j}, e_{m}\right\rangle\right|^{p} \leq C_{\delta} \sum_{m} \widehat{\mu}_{r}\left(b_{m}\right)^{p}<\infty .
$$

Since $T, E \in S_{p}$, we also have $D \in S_{p}$ with

$$
\|D\|_{p}^{p} \geq C_{1} \sum_{m} \widehat{\mu}_{r}\left(b_{m}\right)^{p}
$$

by (4.3) and (4.8). Thus the triangle inequality $\|D\|_{p}^{p}-\|E\|_{p}^{p} \leq\|T\|_{p}^{p}$ for $0<p<1$ yields

$$
\left(C_{1}-C_{\delta}\right) \sum_{m} \widehat{\mu}_{r}\left(b_{m}\right)^{p} \leq\|T\|_{p}^{p} \leq\|A\|^{2 p}\left\|T_{\mu}\right\|_{p}^{p} .
$$

Now, choosing $\delta$ sufficiently close to 1 so that $C_{\delta}<C_{1}$ and then applying the above to each of the $N$ subsequences $\left\{b_{m}\right\}$, we conclude that

$$
\sum_{m} \widehat{\mu}_{r}\left(a_{m}\right)^{p} \leq \frac{N}{C_{1}-C_{\delta}}\|A\|^{2 p}\left\|T_{\mu}\right\|_{p}^{p} .
$$

This estimate holds for all $\mu$ with compact support and thus for arbitrary $\mu$ by an approximation argument. The proof of Theorem $4.5(\mathrm{a}) \Rightarrow(\mathrm{b})$ is complete.

Proof of $(\mathrm{b}) \Rightarrow(\mathrm{a})$. Assume (b). The proof is quite similar to that of (4.4). Fix a large integer $k>0$ such that

$$
k>\frac{n-1}{2 p}-\frac{n}{2} .
$$

By Corollary 3.11 again, we may assume $r<\min \left\{\delta_{k}, 1 / 2\right\}$, where $\delta_{k}$ is the constant as in Lemma 4.3(b). Let $\left\{e_{m}\right\}$ be an arbitrary orthonormal basis for $b^{2}$ and let $A=Q_{k} J$ as in the proof of (a) $\Rightarrow(\mathrm{b})$. But this time the operator $Q_{k}$ is associated with the sequence $\left\{a_{m}\right\}$. Put $h_{m}=A e_{m}=$ $\left(1-\left|a_{m}\right|^{2}\right)^{k+n / 2} R_{k}\left(\cdot, a_{m}\right)$ as before. Let $T=A^{*} T_{\mu} A$. Note that $A$ is bounded and surjective on $b^{2}$ by Lemma 4.3(b). Thus there exists a bounded right inverse of $A$, say $B$, so that $T_{\mu}=B^{*} T B$. It follows that $T_{\mu} \in S_{p}$ if and only if $T \in S_{p}$. We now prove $T \in S_{p}$ in the rest of the proof.

Since $\widehat{\mu}_{r}\left(a_{m}\right) \rightarrow 0$ as $m \rightarrow \infty$, we have $\widetilde{\mu} \in L_{0}$ by Proposition 3.10 and thus $T_{\mu}$ is compact by [7, Theorem 3.12]. So $T$ is also compact. Note $T \geq 0$. Therefore, in order to prove $T \in S_{p}$, it is sufficient to show that

$$
\sum_{m}\left\langle T e_{m}, e_{m}\right\rangle^{p} \leq C \sum_{j} \widehat{\mu}_{r}\left(a_{j}\right)^{p}
$$

for some constant $C=C(n, p, r)$ by Lemma 4.2. To prove (4.9), we first 
note (as in (4.2)) that

$$
\left\langle T e_{m}, e_{m}\right\rangle^{p}=\left\{\int_{B}\left|h_{m}\right|^{2} d \mu\right\}^{p} \leq \sum_{j}\left\{\int_{E_{r}\left(a_{j}\right)}\left|h_{m}\right|^{2} d \mu\right\}^{p}
$$

for each $m$, because $0<p<1$ and $\left\{E_{r}\left(a_{j}\right)\right\}$ is a covering of $B$. Let $m$ and $j$ be given. By (2.6) and Lemma 4.4, we have

$$
\left|h_{m}(x)\right|^{2 p} \lesssim\left\{\frac{\left(1-\left|a_{m}\right|^{2}\right)^{n+2 k}}{\left[x, a_{m}\right]^{2 n+2 k}}\right\}^{p} \approx \int_{E_{r}\left(a_{m}\right)}\left\{\frac{\left(1-|y|^{2}\right)^{n+2 k}}{\left[a_{j}, y\right]^{2 n+2 k}}\right\}^{p} d \lambda(y)
$$

for all $x \in E_{r}\left(a_{j}\right)$. It follows that

$$
\left\langle T e_{m}, e_{m}\right\rangle^{p} \lesssim \sum_{j} \mu\left[E_{r}\left(a_{j}\right)\right]^{p} \int_{E_{r}\left(a_{m}\right)}\left\{\frac{\left(1-|y|^{2}\right)^{n+2 k}}{\left[a_{j}, y\right]^{2 n+2 k}}\right\}^{p} d \lambda(y)
$$

for all $m$. Since $\mu\left[E_{r}\left(a_{j}\right)\right] \approx\left(1-\left|a_{j}\right|^{2}\right)^{n} \widehat{\mu}_{r}\left(a_{j}\right)$, summing up both sides of the above over all $m$ and then changing the order of summation, we obtain

$$
\sum_{m}\left\langle T e_{m}, e_{m}\right\rangle^{p} \lesssim \sum_{j}\left(1-\left|a_{j}\right|^{2}\right)^{n p} \widehat{\mu}_{r}\left(a_{j}\right)^{p} I_{j}
$$

where

$$
I_{j}=\sum_{m} \int_{E_{r}\left(a_{m}\right)}\left\{\frac{\left(1-|y|^{2}\right)^{n+2 k}}{\left[a_{j}, y\right]^{2 n+2 k}}\right\}^{p} d \lambda(y) .
$$

Now, since $p(n+2 k)-n>-1$ by our choice of $k$, we obtain, by Lemma 3.2 (with $\alpha=1$ ) and Lemma 2.5,

$$
I_{j} \lesssim N \int_{B}\left\{\frac{\left(1-|y|^{2}\right)^{n+2 k}}{\left[a_{j}, y\right]^{2 n+2 k}}\right\}^{p} d \lambda(y) \approx\left(1-\left|a_{j}\right|^{2}\right)^{-n p}
$$

for all $j$, where $N=N(1, r)$ is the number provided by Lemma 3.2. Hence, combining this estimate with (4.10), we deduce (4.9). The proof of Theorem 4.5 is complete.

5. Examples. In this section we show that the parameter range (3.8) is sharp. Throughout the section we consider arbitrary $0<p \leq \infty$ and $\alpha$ real, unless otherwise specified.

We first recall the following fact:

If $p \leq-(\alpha+1) / n$ or $p \leq 1+\alpha$, then there exists some $f \geq 0$ such that $f \in L_{\alpha}^{p}$ but $\widetilde{f} \notin L_{\alpha}^{p}$.

The above fact is implicit in [3, Examples 4.8 and 4.9]. Moreover, the proofs in [3] show that the example of function $f$ above also satisfies $\widehat{f}_{r} \in L_{\alpha}^{p}$ 
for each $r \in(0,1)$. Note that the above fact takes care of an example we need for the case $\alpha \geq 0$. For $\alpha<0$, note that the parameter range for which we need an example in (3.8) reduces as follows:

$$
p \leq 1+\alpha / n .
$$

Given $\delta>1$, let $\Gamma_{\delta}$ be the nontangential approach region with vertex $\mathbf{e}:=(1,0, \ldots, 0)$ consisting of all points $x \in B$ such that

$$
|x-\mathbf{e}|<\delta(1-|x|) .
$$

In case $\alpha<0$, certain radial functions restricted to nontangential approach regions will be our examples. To estimate such functions we need positivity of the kernel function as in the next lemma.

Lemma 5.1. There exist some $\delta>1$ and a constant $C>0$ such that

$$
R(x, y) \geq C /[x, y]^{n}
$$

for $x, y \in \Gamma_{\delta}$ with $|x|,|y| \geq 1 / \sqrt{2}$.

Proof. Let $x, y \in \Gamma_{\delta}$, where $\delta>1$ is to be chosen later. Assume $|x|,|y| \geq$ $1 / \sqrt{2}$. Taking $\delta$ sufficiently close to 1 , we may assume $x_{1}, y_{1}>0$. Let $x^{\prime}=$ $\left(0, x_{2}, \ldots, x_{n}\right)$. Since $x \in \Gamma_{\delta}$, we have

$$
\left|x^{\prime}\right|^{2}+(1-|x|)^{2} \leq\left|x^{\prime}\right|^{2}+\left(1-x_{1}\right)^{2}=|x-\mathbf{e}|^{2}<\delta^{2}(1-|x|)^{2}
$$

and thus $\left|x^{\prime}\right| /|x|<\varepsilon(1-|x|)$, where $\varepsilon=\sqrt{2\left(\delta^{2}-1\right)}$. Hence

$$
\frac{\left|x^{\prime}\right|\left|y^{\prime}\right|}{|x||y|}<\varepsilon^{2}(1-|x|)(1-|y|) \leq \varepsilon^{2}(1-|x||y|)^{2}
$$

and

$$
\begin{aligned}
1-\frac{x_{1} y_{1}}{|x||y|} & =1-\left(1-\frac{\left|x^{\prime}\right|^{2}}{|x|^{2}}\right)^{1 / 2}\left(1-\frac{\left|y^{\prime}\right|^{2}}{|y|^{2}}\right)^{1 / 2} \\
& \leq 1-\left(1-\frac{\left|x^{\prime}\right|^{2}}{|x|^{2}}\right)\left(1-\frac{\left|y^{\prime}\right|^{2}}{|y|^{2}}\right) \\
& <\frac{\left|x^{\prime}\right|^{2}}{|x|^{2}}+\frac{\left|y^{\prime}\right|^{2}}{|y|^{2}} \leq \varepsilon^{2}\left[(1-|x|)^{2}+(1-|y|)^{2}\right] \leq 2 \varepsilon^{2}(1-|x||y|)^{2} .
\end{aligned}
$$

Combining these inequalities, we obtain

$$
1-\frac{x \cdot y}{|x||y|} \leq 1-\frac{x_{1} y_{1}}{|x||y|}+\frac{\left|x^{\prime}\right|\left|y^{\prime}\right|}{|x||y|} \leq 3 \varepsilon^{2}(1-|x||y|)^{2}
$$

and thus

$$
[x, y]^{2}=(1-|x||y|)^{2}+2(|x||y|-x \cdot y) \leq\left(1+6 \varepsilon^{2}\right)(1-|x||y|)^{2} .
$$


Therefore, we conclude that

$$
\left(\frac{1-|x|^{2}|y|^{2}}{[x, y]}\right)^{2}-\frac{4|x|^{2}|y|^{2}}{n}>\frac{(1+|x||y|)^{2}}{\left(1+6 \varepsilon^{2}\right)}-\frac{4}{n} \geq \frac{9 / 4}{\left(1+6 \varepsilon^{2}\right)}-2>0,
$$

if $\varepsilon$ is sufficiently small. This, together with (1.2), implies the lemma.

Now, given $\gamma \geq 0$, let $f_{\gamma}$ be the function on $B$ defined by

$$
f_{\gamma}(x)=\frac{1}{(1-|x|)^{n}}\left(\log \frac{2}{1-|x|}\right)^{-\gamma} .
$$

It is clear from Lemma 2.1 that $\widehat{\left(f_{\gamma}\right)_{r}} \approx f_{\gamma}$ for each $r \in(0,1)$.

LEMMA 5.2. Given $\delta>1$ and $\gamma \geq 0$, the function $g=f_{\gamma} \chi_{\Gamma_{\delta}}$ has the following properties:

(a) $g \in L_{\alpha}^{p}$ if and only if one of the following conditions holds:

(i) $p<1+\alpha / n$;

(ii) $p=1+\alpha / n$ and $\gamma>1 / p$.

(b) Given $r \in(0,1)$, we have $f_{\gamma} \chi_{\Gamma_{\delta_{1}}} \lesssim \widehat{g}_{r} \lesssim f_{\gamma} \chi_{\Gamma_{\delta_{2}}}$ for some $\delta_{1}$ and $\delta_{2}$.

(c) If $\gamma>1$, then $\widetilde{g} \gtrsim f_{\gamma-1} \chi_{\Gamma_{\delta_{3}}}$ for some $\delta_{3}$.

(d) If $\gamma \leq 1$, then $\widetilde{g}=\infty$ on some open set.

Proof. Fix $\delta>1 / 2$ and $\gamma \geq 0$. Since $1-|x| \approx|x-\mathbf{e}|$ for $x \in \Gamma_{\delta}$, we have

$$
\int_{B}|g|^{p} d V_{\alpha} \approx \int_{\Gamma_{\delta}} \frac{1}{|x-\mathbf{e}|^{p n-\alpha}}\left(\log \frac{2}{|x-\mathbf{e}|}\right)^{-p \gamma} d x
$$

Integrating the last integral in polar coordinates, we have (a).

Now we prove (b). Fix $r \in(0,1)$. Put $\delta_{1}=(1+\delta) / 2$ and pick a sufficiently small radius $s$ in such a way that $\varepsilon:=\left(s+\delta_{1}\right)(1+s) /(1-s)<\delta$. For $x \in \Gamma_{\delta_{1}}$ and $y \in E_{s}(x)$, by Lemmas 2.1 and 2.2 we have

$$
|y-\mathbf{e}| \leq|x-y|+|x-\mathbf{e}|<s[x, y]+\delta_{1}(1-|x|) \leq \varepsilon(1-|y|) .
$$

So, $\Gamma_{\delta} \cap E_{s}(x)=E_{s}(x)$ for $x \in \Gamma_{\delta_{1}}$. Thus $\widehat{g}_{r} \geq \widehat{\left(f_{\gamma}\right)_{r}} \chi_{\Gamma_{\delta_{1}}} \approx f_{\gamma} \chi_{\Gamma_{\delta_{1}}}$. A similar argument shows $\Gamma_{\delta} \cap E_{r}(x)=\emptyset$ for $x \notin \Gamma_{\delta_{2}}$, where $\delta_{2}=(r+\delta)(1+r) /(1-r)$. Thus $\widehat{g}_{r} \leq \widehat{\left(f_{\gamma}\right)_{r}} \chi_{\Gamma_{\delta_{2}}} \approx f_{\gamma} \chi_{\Gamma_{\delta_{2}}}$.

Finally, we show (c) and (d). We may assume that $\delta$ is sufficiently close to 1 so that Lemma 5.1 is available. Assume $x \in \Gamma_{\delta}$ for the rest of the proof. We may further assume $|x| \geq 1 / \sqrt{2}$. Since $1-|x||y|=1-|x|+|x|(1-|y|) \leq$ $2(1-|x|)$ for $|y| \geq|x|$, by Lemma 5.1 and (5.2) we have

$$
|R(x, y)|^{2} \gtrsim \frac{1}{[x, y]^{2 n}} \gtrsim \frac{1}{(1-|x||y|)^{2 n}} \approx \frac{1}{(1-|x|)^{2 n}}
$$


for $y \in \Gamma_{\delta}$ with $|y-\mathbf{e}| \leq 1-|x|$. Thus

$$
\begin{aligned}
\widetilde{g}(x) & \gtrsim(1-|x|)^{-n} \int_{\Gamma_{\delta} \cap\{|y-\mathbf{e}| \leq 1-|x|\}} f_{\gamma}(y) d y \\
& \approx(1-|x|)^{-n} \int_{\Gamma_{\delta} \cap\{|y-\mathbf{e}| \leq 1-|x|\}} \frac{1}{|y-\mathbf{e}|^{n}}\left(\log \frac{2}{|y-\mathbf{e}|}\right)^{-\gamma} d x \\
& \approx(1-|x|)^{-n} \int_{0}^{1-|x|} \frac{1}{t}\left(\log \frac{2}{t}\right)^{-\gamma} d t \\
& \approx f_{\gamma-1}(x) \quad \text { if } \gamma>1 .
\end{aligned}
$$

So, (c) holds. Also, the last integral above diverges for $\gamma \leq 1$ and thus (d) holds.

Now, using Lemma 5.2, we have examples for the remaining parameters in (5.1) as follows.

ExAmple 5.3. Let $\gamma \geq 0$ and $\delta>1$. Put $g=f_{\gamma} \chi_{\Gamma_{\delta}}$. Assume $0<p \leq$ $1+\alpha / n$. Then the following statements hold for each $r \in(0,1)$ :

(a) If $p<1+\alpha / n$ and $\gamma \leq 1$, then $\widehat{g}_{r} \in L_{\alpha}^{p}$ and $\widetilde{g}=\infty$ on some open set.

(b) If $p=1+\alpha / n$ and $\max \{1,1 / p\}<\gamma \leq 1+1 / p$, then $\widehat{g}_{r} \in L_{\alpha}^{p}$ but $\widetilde{g} \notin L_{\alpha}^{p}$.

\section{References}

[1] L. V. Ahlfors, Möbius transformations in several dimensions, Univ. of Minnesota, Minneapolis, 1981.

[2] S. Axler, P. Bourdon and W. Ramey, Harmonic Function Theory, Springer, New York, 1992.

[3] B. R. Choe, Note on the Berezin transform on Herz spaces, RIMS Kyokuroku 1519 (2006), 21-37.

[4] B. R. Choe, H. Koo and Y. J. Lee, Positive Schatten(-Herz) class Toeplitz operators on the half-space, Potential Anal. 27 (2007), 73-100.

[5] B. R. Choe, H. Koo and K. Na, Positive Toeplitz operators of Schatten-Herz type, Nagoya Math. J. 185 (2007), 31-62.

[6] B. R. Choe and Y. J. Lee, Note on atomic decompositions of harmonic Bergman functions, in: Complex Analysis and its Applications, OCAMI Stud. Ser. 2, Osaka Municipal Univ. Press, 2007, 11-24.

[7] B. R. Choe, Y. J. Lee and K. Na, Toeplitz operators on harmonic Bergman spaces, Nagoya Math. J. 174 (2004), 165-186.

[8] R. R. Coifman and R. Rochberg, Representation theorems for holomorphic and harmonic functions in $L^{p}$, Astérisque 77 (1980), 11-66.

[9] D. Luecking, Trace ideal criteria for Toeplitz operators, J. Funct. Anal. 73 (1987), $345-368$. 
[10] D. Luecking and K. Zhu, Composition operators belonging to Schatten classes, Amer. J. Math. 114 (1992), 1127-1145.

[11] J. Miao, Reproducing kernels for harmonic Bergman spaces of the unit ball, Monatsh. Math. 125 (1998), 25-35.

[12] —, Toeplitz operators on harmonic Bergman spaces, Integral Equations Operator Theory 27 (1997), 426-438.

[13] G. Ren and U. Kähler, Boundary behavior of Gleason's problem in hyperbolic harmonic Bergman spaces, Sci. China Ser. A Math. 48 (2005), 145-154.

[14] K. Zhu, Operator Theory in Function Spaces, Dekker, New York, 1989.

[15] —, Schatten class Toeplitz operators on the weighted Bergman spaces of the unit ball, New York J. Math. 13 (2007), 299-316.

Department of Mathematics

Korea University

Seoul 136-713, Korea

E-mail: cbr@korea.ac.kr

koohw@korea.ac.kr
Department of Mathematics Chonnam National University Gwangju 500-757, Korea E-mail: leeyj@chonnam.ac.kr

Received December 29, 2007

Revised version June 19, 2008 